\title{
Upper Bounds for the Determinant of a Row Stochastic Matrix
}

\author{
Karl Goldberg \\ Institute for Basic Standards, National Bureau of Standards, Washington, D.C.
}

(February 16, 1966)

\begin{abstract}
If $A=\left(a_{i j}\right)$ is a row stochastic matrix of order $n$ then $|\operatorname{det} A| \leqslant\left(1-\sum_{j=1}^{n} \min _{i} a_{i j}\right)^{n-1}$. If $\alpha$ is a 1 by $n$ real vector such that every element of $\alpha A$ is nonnegative then $|\operatorname{det} A| \leqslant s(\alpha) /|g(\alpha)|$, where $s(\alpha)$ is the sum of the elements of $\alpha$ and $g(\alpha)$ is the element of $\alpha$ of greatest absolute value. The conditions for equality are determined in both cases.
\end{abstract}

Key Words: Determinants, matrices (row stochastic), upper bounds.

It is known that if $\mathrm{A}$ is a row stochastic matrix then $|\operatorname{det} \mathbf{A}| \leqslant 1$ with equality if and only if $\mathbf{A}$ is a permutation matrix. We will use this result to extend itself.

The result is equivalent to the following statement about nonnegative matrices. ${ }^{1}$ Let $A$ be a nonnegative matrix of order $n$, let $r_{A}^{(i)}$ denote the sum of the elements in the $i$ th row, let

$$
r_{A}=r_{A}^{(1)} r_{A}^{(2)} \ldots r_{A}^{(n)}
$$

and

$$
R_{A}=\operatorname{diag}\left(r_{A}^{(1)}, r_{A}^{(2)}, \ldots, r_{A}^{(n)}\right) \text {. }
$$

Note that $r_{A}=0$ if and only if some row of $A$ is all 0 's. Otherwise $R_{A}^{-1} A$ is row stochastic. Thus $|\operatorname{det} \mathrm{A}| \leqslant \mathrm{r}_{\mathrm{A}}$ with equality if and only if either $\mathrm{r}_{\mathrm{A}}=0$ or $\mathrm{A}=\mathrm{R}_{\mathrm{A}} \mathrm{P}$ for some permutation matrix $\mathrm{P}$.

We use the following procedure. Given a row stochastic matrix $A$, let $B$ be a nonsingular matrix such that $A B \geqslant 0$ (i.e., $A B$ is nonnegative). Then the above result implies that

$$
|\operatorname{det} A| \leqslant \frac{r_{A B}}{|\operatorname{det} B|}
$$

with equality if and only if either $r_{A B}=0$ or $A=R_{A B} P B^{-1}$. The same procedure with $C A \geqslant 0$ yields a similar result. Since $B$ (or $C$ ) need not be nonnegative we have room for improvement.

From this point on $A$ will denote a row stochastic matrix of order $n$.

Lower case Greek letters will denote 1 by $n$ real vectors including

$$
\epsilon=\left(\begin{array}{llll}
1 & 1 & \ldots & \ldots
\end{array}\right) \text { and } \delta_{i}=\left(\begin{array}{llllll}
0 & \ldots & 0 & 0 & 0 & \ldots
\end{array}\right)=\left(\delta_{i j}\right) .
$$

${ }^{1} \mathrm{~A}$ matrix is nonnegative if each element is real and nonnegative. It is row stochastic if, in addition, each row sum equals 1 .
For a vector $\alpha, s(\alpha)$ will denote the sum of its elements, $g(\alpha)$ will denote its element of greatest absolute value, and $M_{i}(\alpha)$ will denote the matrix

$$
M_{i}(\alpha)=\left[\begin{array}{l}
\delta_{1} \\
\cdot \\
\cdot \\
\delta_{i-1} \\
\alpha \\
\delta_{i+1} \\
\cdot \\
\cdot \\
\cdot \\
\delta_{n}
\end{array}\right]
$$

i.e., the identity matrix with the $i$ th row replaced by $\alpha$. For future clarity we note now that

$$
s(\alpha)=\alpha \epsilon^{T},
$$

while $\epsilon^{T} \alpha$ is the matrix

$$
\epsilon^{T} \alpha=\left[\begin{array}{c}
\alpha \\
\alpha \\
\cdot \\
\cdot \\
\cdot \\
\alpha
\end{array}\right]
$$

Finally $A \epsilon^{T}=\epsilon^{T}$. We shall prove:

THEOREM 1. Let A be a row stochastic matrix of order $\mathrm{n}$, and let

$$
\mathrm{c}=\sum_{\mathrm{j}=1}^{\mathrm{n}} \min _{i} \mathrm{a}_{\mathrm{ij}} .
$$

Then

$$
|\operatorname{det} \mathrm{A}| \leqslant(1-\mathrm{c})^{\mathrm{n}-1}
$$


with equality if and only if

$$
\mathrm{A}=(1-\mathrm{s}(\beta)) \mathrm{P}+\epsilon^{\mathrm{T}} \beta
$$

for some vector $\beta \geqslant 0$ with $1 \geqslant \mathrm{~s}(\beta)$, and some permutation matrix $\mathrm{P}$.

THEOREM 2. Let A be a row stochastic matrix and. $\alpha$ a real, nonzero vector such that $\alpha \mathrm{A} \geqslant 0$. Then

$$
|\operatorname{det} \mathrm{A}| \leqslant \frac{\mathrm{s}(\alpha)}{|\mathrm{g}(\alpha)|}
$$

with equality if and only if either $\mathrm{s}(\alpha)=0$, or $\alpha$ has exactly one positive element (in the i-th position) and

$$
\mathrm{A}=\mathrm{M}_{\mathrm{i}}(\gamma) \mathrm{P}
$$

for some permutation matrix $\mathrm{P}$, with

$$
\gamma=\frac{1}{\mathrm{~g}(\alpha)}\left\{(\mathrm{g}(\alpha)+\mathrm{s}(\alpha)) \delta_{\mathrm{i}}-\alpha\right\}
$$

To prove Theorem 1 let $c_{j}=\min _{i} a_{i j}$ and let

$$
B=I-\sum_{j=1}^{n} c_{j} \epsilon^{T} \delta_{j}
$$

The matrix $\epsilon^{T} \delta_{j}$ has l's in the $j$ th column and 0 's elsewhere, so that $A B=\left(a_{i j}-c_{j}\right)$. By the definition of the $c_{j}, A B$ is nonnegative.

The row sums of $\mathrm{AB}$ are the elements of the vector

$$
A B \epsilon^{T}=A \epsilon^{T}-\sum_{j=1}^{n} c_{j} \epsilon^{T} \delta_{j} \epsilon^{T}=(1-c) \epsilon^{T} .
$$

Thus $r_{A B}^{(i)}=1-c$ for all $i$, so that

$$
r_{A B}=(1-c)^{n} .
$$

It is a simple exercise (left to the reader) to prove that

and

$$
\operatorname{det} B=1-c
$$

$$
B^{-1}=I+(1-c)^{-1} \sum_{j=1}^{n} c_{j} \epsilon^{T} \delta_{j} \quad c \neq 1 .
$$

If $c \neq 1$ then, from our previous discussion, the necessity case of Theorem 1 follows with

$$
\beta=\left(c_{1} c_{2} \ldots c_{n}\right) .
$$

In the case of sufficiency, if $A=(1-s(\beta)) P+\epsilon^{T} \beta$ then all the row sums of $A$ equal $\mathrm{l}\left(A \epsilon^{T}=\epsilon^{T}\right), A$ is nonnegative if $\beta \geqslant 0$, and $1 \geqslant s(\beta), \beta=\left(c_{1} c_{2} \ldots c_{n}\right)$ with the $c_{j}$ defined as above, and $|\operatorname{det} A|=(1-s(\beta))^{n-1}$.

A short discussion of the value of $c$ will complete our proof. Since $A$ is row stochastic the sum of all of its elements is $n$. Thus

$$
n=\sum_{j=1}^{n} \sum_{i=1}^{n} a_{i j} \geqslant \sum_{j=1}^{n} \sum_{i=1}^{n} c_{j}=n c
$$

with equality $(c=1)$ if and only if $a_{i j}=c_{j}$ for all $i, j$, in which case $A$ is of rank 1 . Then $\operatorname{det} A=1$ for $n$ $=1$, and $\operatorname{det} A=0$ with $n>1$, and $A=\epsilon^{T} \beta$. This checks with the statement of Theorem 1 and completes our proof.

To prove Theorem 2 let $C=M_{i}(\alpha)$. The rows of $C A$ are the rows of $A$ except for the $i$ th row which is $\alpha A$. By hypothesis $\alpha A \geqslant 0$ so that $C A$ is nonnegative.

The row sums of $C A$ are 1 except for the $i$ th row whose sum is

$$
s(\alpha A)=(\alpha A) \epsilon^{T}=\alpha\left(A \epsilon^{T}\right)=\alpha \epsilon^{T}=s(\alpha) .
$$

Thus $r_{C A}=s(\alpha)$ and $R_{C A}$ is the identity matrix transformed by replacing the 1 in the $i$ th position on the diagonal by $s(\alpha)$. Note that, if $s(\alpha) \neq 0$, we have

$$
R_{C A}^{-1} M_{i}(\alpha)=M_{i}(\alpha / s(\alpha)) .
$$

The determinant of $C$ is the product of its diagonal elements. Let

$$
\alpha=\left(a_{1} a_{2} . \quad . a_{n}\right) .
$$

Then $\operatorname{det} C=a_{i}$. By our previous discussion $|\operatorname{det} A|$ $\leqslant r_{C A} /|\operatorname{det} C|$. Thus

$$
|\operatorname{det} A| \leqslant \frac{s(\alpha)}{\left|a_{i}\right|}
$$

This inequality holds for all $i$; the best of the inequalities is

$$
|\operatorname{det} A| \leqslant \frac{s(\alpha)}{|g(\alpha)|}
$$

Suppose $g(\alpha)=a_{i}$. We have equality if and only if either $s(\alpha)=0$, (whence $\alpha A=0, \alpha \neq 0$, so that det $A=0)$ or, if $s(\alpha) \neq 0$ :

$$
A=C^{-1} R_{C A} P=\left(R_{C A}^{-1} M_{i}(\alpha)\right)^{-1} P=\left(M_{i}(\alpha / s(\alpha))^{-1} P\right.
$$

for some permutation matrix $P$.

But $\left(M_{i}(\alpha / s(\alpha))\right)^{-1}=M_{i}(\gamma)$ with

$$
\gamma=a_{i}^{-1}\left(-a_{i} \ldots-a_{i-1} s(\alpha)-a_{i+1} \ldots .-a_{n}\right) .
$$

Since $A=M_{i}(\gamma) P$ is nonnegative we must have $a_{j} \leqslant 0$ for $j \neq i$. This completes the proof of Theorem 2 .

(Paper 70B2-178) 